\title{
SUMS OF DEPENDENT NONNEGATIVE RANDOM VARIABLES WITH SUBEXPONENTIAL TAILS
}

\author{
BANGWON KO* AND \\ QIHE TANG, ${ }^{* * *}$ The University of Iowa
}

\begin{abstract}
In this paper we study the asymptotic tail probabilities of sums of subexponential, nonnegative random variables, which are dependent according to certain general structures with tail independence. The results show that the subexponentiality of the summands eliminates the impact of the dependence on the tail behavior of the sums.
\end{abstract}

Keywords: Asymptotics; copula; dependence; subexponentiality; uniformity

2000 Mathematics Subject Classification: Primary 62E20

Secondary 60G70; 62H20

\section{Introduction}

We are interested in the tail probabilities of sums of dependent and heavy-tailed random variables. Throughout this paper, by saying that a distribution $F$ is concentrated on $[0, \infty)$, we mean that $F(0-)=0$ and $\bar{F}(x)=1-F(x)>0$ for all $x>0$. One of the most important classes of heavy-tailed distributions is the subexponential class, written as $\&$. By definition, a distribution $F$ concentrated on $[0, \infty)$ belongs to the class $\&$ if the relation

$$
\lim _{x \rightarrow \infty} \frac{\overline{F^{* n}}(x)}{\bar{F}(x)}=n
$$

holds for some (or, equivalently, for all) $n=2,3, \ldots$, where $F^{* n}$ denotes the $n$-fold convolution of $F$. Hence, if $X_{1}, X_{2}, \ldots$ are independent and identically distributed (i.i.d.) random variables with common distribution $F \in \delta$ then, for every $n=2,3, \ldots$,

$$
\mathrm{P}\left(S_{n}>x\right) \sim \mathrm{P}\left(X_{(n)}>x\right) \sim n \bar{F}(x) .
$$

Here and henceforth, $S_{n}=X_{1}+\cdots+X_{n}, X_{(n)}=\max \left\{X_{1}, \ldots, X_{n}\right\}$, all limit relationships are for $x$ tending to $\infty$ unless stated otherwise, and $a(x) \sim b(x)$ stands for $\lim a(x) / b(x)=1$.

We also need two other classes of heavy-tailed distributions. A distribution $F$ is said to be long tailed, written as $F \in \mathcal{L}$, if the relation

$$
\lim _{x \rightarrow \infty} \frac{\bar{F}(x-t)}{\bar{F}(x)}=1
$$

holds for some (or, equivalently, for all) $t>0$, and $F$ is said to be dominatedly varying tailed, written as $F \in \mathcal{D}$, if the relation

$$
\limsup _{x \rightarrow \infty} \frac{\bar{F}(t x)}{\bar{F}(x)}<\infty
$$

Received 30 July 2007; revision received 6 December 2007.

* Postal address: Department of Statistics and Actuarial Science, The University of Iowa, 241 Schaeffer Hall, Iowa City, IA 52242, USA.

** Email address: qtang@ stat.uiowa.edu 
holds for some (or, equivalently, for all) $0<t<1$. It is well known that $\mathscr{D} \cap \mathcal{L} \subset \& \subset \mathcal{L}$; see, e.g. Embrechts et al. (1997, Section 1.4).

Because the class $\&$ enjoys the nice asymptotic property in (1.2), it has been extensively applied to modeling heavy-tailed distributions appearing in insurance and finance. However, owing to the very definition (1.1), the mainstream study of subexponentiality has been restricted to the i.i.d. case. This seriously limits the usefulness of the beautiful theory of subexponentiality in practice. The purpose of this paper is to find out to what extent $X_{1}, \ldots, X_{n}$ can be dependent while relations (1.2) remain, or, in other words, how insensitive the tail behavior of the sum $S_{n}$ is on the dependence among its subexponential summands.

The insensitivity of subexponentiality has recently been observed by different researchers. For $F \in \mathscr{D} \cap \mathcal{L}$, Geluk and $\mathrm{Ng}$ (2006) proved relations (1.2) under the assumption that the random variables $X_{1}, \ldots, X_{n}$ are negatively associated, meaning that, for every pair of disjoint subsets $I$ and $J$ of $\{1, \ldots, n\}$, the inequality $\operatorname{cov}\left\{f\left(X_{i}, i \in I\right), g\left(X_{j}, j \in J\right)\right\} \leq 0$ holds for all coordinatewise increasing functions $f$ and $g$ for which the covariance exists. Tang (2008) relaxed the dependence structure from negative association to pairwise negative quadrant dependence; see Remark 2.4, below, for the definition. Albrecher et al. (2006) studied the problem for $n=2$ using copulas to describe the dependence structure of $X_{1}$ and $X_{2}$. Their Lemma 2.7 showed that relations (1.2) hold if $F$ is absolutely continuous and the copula density of $\left(X_{1}, X_{2}\right)$ exists and is uniformly bounded on $[c, 1]^{2}$ for some $0<c<1$; see also Remark 2.3, below, for details. Tang and Tsitsiashvili (2003) observed the insensitivity of subexponentiality from a different angle. Consider the weighted random variables $X_{1}=$ $\omega_{1} Y_{1}, \ldots, X_{n}=\omega_{n} Y_{n}$, where the primary random variables $Y_{1}, \ldots, Y_{n}$ are i.i.d. with common subexponential distribution while the random weights $\omega_{1}, \ldots, \omega_{n}$, independent of the primary random variables, are dependent with common distribution concentrated on $[a, b]$ for some $0<a<b<\infty$. For this case, relations (1.2) still hold by Theorem 3.1 of Tang and Tsitsiashvili (2003). All these works reveal a phenomenon that, when deriving the tail asymptotics for the sum of dependent random variables, the subexponentiality assumption eliminates the impact of certain dependence structures among the summands.

In this paper we shall propose another general dependence structure, which does not require the existence of probability densities or copula densities and which allows both positive and negative dependence to a certain extent. Our discussions in Section 2.1, below, show that our assumption is satisfied by many commonly used dependence structures.

The rest of this paper consists of two sections. Section 2 is devoted to the bivariate case under our basic assumption on the dependence between $X_{1}$ and $X_{2}$, while Section 3 extends the scenario to the multivariate case under a modified version of the dependence assumption.

\section{The bivariate case}

\subsection{Our assumption on the dependence}

In this section we study the case in which $n=2$ with $X_{1}$ and $X_{2}$ distributed by $F_{1}$ and $F_{2}$ concentrated on $[0, \infty)$, respectively. Our basic assumption is as follows.

Assumption 2.1. For $(i, j)=(1,2)$ or $(2,1)$, the relation

$$
\frac{\mathrm{P}\left(X_{j}>x-t \mid X_{i}=t\right)}{\mathrm{P}\left(X_{j}>x-t\right)}=O(1)
$$

holds uniformly for all $t \in\left[x_{0}, x\right]$ for some large $x_{0}>0$. 
Here the uniformity means that

$$
\limsup _{x \rightarrow \infty} \sup _{x_{0} \leq t \leq x} \frac{\mathrm{P}\left(X_{j}>x-t \mid X_{i}=t\right)}{\mathrm{P}\left(X_{j}>x-t\right)}<\infty .
$$

When $t$ is not a possible value of $X_{i}$, i.e. $\mathrm{P}\left(X_{i} \in \Delta\right)=0$ for some open interval $\Delta$ containing $t$, the conditional probability in Assumption 2.1 is simply understood as 0 . Since

$$
\sup _{x-x_{0}<t \leq x} \frac{\mathrm{P}\left(X_{j}>x-t \mid X_{i}=t\right)}{\mathrm{P}\left(X_{j}>x-t\right)} \leq \frac{1}{\overline{F_{j}}\left(x_{0}\right)},
$$

relation (2.1) is equivalent to

$$
\limsup _{x \rightarrow \infty} \sup _{x_{0} \leq t \leq x-x_{0}} \frac{\mathrm{P}\left(X_{j}>x-t \mid X_{i}=t\right)}{\mathrm{P}\left(X_{j}>x-t\right)}<\infty .
$$

Intuitively, Assumption 2.1 requires that the dependence structure between $X_{1}$ and $X_{2}$ should not be too positive. Hence, it excludes extremely positive dependence structures such as comonotonicity. However, Assumption 2.1 can still be satisfied by most of the extremely negative dependence structures such as countermonotonicity. As an example, if $X_{1} X_{2}=1$ then relation (2.2) holds with $x_{0}=1$.

In the following remarks we show that Assumption 2.1 indeed allows a wide range of dependence structures. We only consider Assumption 2.1 with $(i, j)=(1,2)$.

Remark 2.1. We show that Assumption 2.1 is satisfied if $X_{2}$ is stochastically decreasing in $X_{1}$, written as $\operatorname{SD}\left(X_{2} \mid X_{1}\right)$, meaning that, for each fixed $x_{2}$, the conditional probability $\mathrm{P}\left(X_{2}>\right.$ $x_{2} \mid X_{1}=x_{1}$ ) is decreasing (not necessarily strictly) in $x_{1}$. Actually, in this case, for every fixed $x_{0} \geq 0$ for which $F_{1}\left(x_{0}\right)>0$, it holds uniformly, for all $t \geq x_{0}$, that

$$
\begin{aligned}
\mathrm{P}\left(X_{2}>x-t \mid X_{1}=t\right) & =\frac{\int_{0-}^{x_{0}} \mathrm{P}\left(X_{2}>x-t \mid X_{1}=t\right) \mathrm{d} F_{1}(u)}{F_{1}\left(x_{0}\right)} \\
& \leq \frac{\int_{0-}^{x_{0}} \mathrm{P}\left(X_{2}>x-t \mid X_{1}=u\right) \mathrm{d} F_{1}(u)}{F_{1}\left(x_{0}\right)} \\
& \leq \frac{\overline{F_{2}}(x-t)}{F_{1}\left(x_{0}\right)} .
\end{aligned}
$$

Hence, Assumption 2.1 is satisfied. We comment that $\operatorname{SD}\left(X_{2} \mid X_{1}\right)$ reveals a flavor of negative dependence. In the literature this dependence structure is also called negative regression dependence; see, e.g. Lehmann (1966).

Remark 2.2. For simplicity, let $F_{1}$ and $F_{2}$ be absolutely continuous, and let $X_{1}$ and $X_{2}$ be dependent according to a copula $C\left(u_{1}, u_{2}\right)$ for $\left(u_{1}, u_{2}\right) \in[0,1]^{2}$. Thus, the joint distribution of $X_{1}$ and $X_{2}$ is given by

$$
H\left(x_{1}, x_{2}\right)=C\left(F_{1}\left(x_{1}\right), F_{2}\left(x_{2}\right)\right) ;
$$

see, e.g. Nelsen (2006, p. 15). Let $U_{1}=F_{1}\left(X_{1}\right)$ and $U_{2}=F_{2}\left(X_{2}\right)$, so that they are two uniform random variables following the joint distribution $C\left(u_{1}, u_{2}\right)$. Under Assumption 2.1, it is not difficult to verify that $X_{1}$ and $X_{2}$ are tail independent in the sense that the (upper) tail dependence measure, defined by

$$
\chi=\lim _{u \rightarrow 1} \mathrm{P}\left(U_{2}>u \mid U_{1}>u\right),
$$


is equal to 0 . Indeed, for all $u$ close to 1 such that $F_{1}^{-1}(u) \geq x_{0}$ and $F_{2}^{-1}(u) \geq x_{0}$,

$$
\begin{aligned}
\mathrm{P}\left(U_{2}>u \mid U_{1}>u\right) & =\int_{F_{1}^{-1}(u)}^{\infty} \frac{\mathrm{P}\left(X_{2}>F_{2}^{-1}(u) \mid X_{1}=t\right)}{1-u} \mathrm{~d} F_{1}(t) \\
& \leq(1-u) \sup _{F_{1}^{-1}(u)<t<\infty} \frac{\mathrm{P}\left(X_{2}>F_{2}^{-1}(u) \mid X_{1}=t\right)}{1-u} \\
& \leq(1-u) \sup _{x_{0} \leq t \leq F_{2}^{-1}(u)+t} \frac{\mathrm{P}\left(X_{2}>F_{2}^{-1}(u)+t-t \mid X_{1}=t\right)}{\mathrm{P}\left(X_{2}>F_{2}^{-1}(u)+t-t\right)} .
\end{aligned}
$$

Using the change of variables $\tilde{x}=F_{2}^{-1}(u)+t$, we find that, as $u \rightarrow 1$,

$$
\mathrm{P}\left(U_{2}>u \mid U_{1}>u\right) \leq(1-u) \sup _{x_{0} \leq t \leq \tilde{x}} \frac{\mathrm{P}\left(X_{2}>\tilde{x}-t \mid X_{1}=t\right)}{\mathrm{P}\left(X_{2}>\tilde{x}-t\right)}=O(1-u) .
$$

Hence, $\chi=0$ as claimed.

To overcome some limitations of the tail dependence measure $\chi$, Coles et al. (1999) defined another tail dependence measure as

$$
\bar{\chi}=\lim _{u \rightarrow 1} \frac{2 \log \mathrm{P}\left(U_{1}>u\right)}{\log \mathrm{P}\left(U_{1}>u, U_{2}>u\right)}-1 .
$$

By (2.3), it is clear that $\bar{\chi} \leq 0$ if it exists.

Remark 2.3. Now we examine Assumption 2.1 from the perspective of copulas. Following the notation of Remark 2.2, further assume that the first-order partial derivative

$$
C_{1}\left(u_{1}, u_{2}\right)=\frac{\partial}{\partial u_{1}} C\left(u_{1}, u_{2}\right)
$$

exists. Then, by the copula representation of the conditional distribution, Assumption 2.1 with $(i, j)=(1,2)$ can be restated as the relation

$$
\frac{1-C_{1}\left(F_{1}(t), F_{2}(x-t)\right)}{\overline{F_{2}}(x-t)}=O(1),
$$

which holds uniformly for all $t \in\left[x_{0}, x\right]$ (or, equivalently, for all $t \in\left[x_{0}, x-x_{0}\right]$ ) for some large $x_{0}>0$. Furthermore, if the second-order mixed partial derivative, usually called the copula density,

$$
C_{12}\left(u_{1}, u_{2}\right)=\frac{\partial^{2}}{\partial u_{1} \partial u_{2}} C\left(u_{1}, u_{2}\right)
$$

exists and is uniformly bounded by some constant $M>0$ for all $\left(u_{1}, u_{2}\right) \in[c, 1]^{2}$ for some $0<c<1$, then Assumption 2.1 is satisfied. To see this, let us choose some $x_{0}>0$ such that $F_{1}\left(x_{0}\right) \geq c$ and $F_{2}\left(x_{0}\right) \geq c$. It holds, for all $t \in\left[x_{0}, x-x_{0}\right]$, that

$$
\frac{\mathrm{P}\left(X_{2}>x-t \mid X_{1}=t\right)}{\mathrm{P}\left(X_{2}>x-t\right)}=\frac{\int_{x-t}^{\infty} C_{12}\left(F_{1}(t), F_{2}(z)\right) \mathrm{d} F_{2}(z)}{\mathrm{P}\left(X_{2}>x-t\right)} \leq M .
$$

Hence, relation (2.2) holds. This boundedness assumption on the copula density $C_{12}\left(u_{1}, u_{2}\right)$ was proposed in Albrecher et al. (2006, Lemma 2.7). Restricting to Archimedean copulas, the verification of Assumption 2.1 becomes even easier by looking at the generator and its first two derivatives, as done in Albrecher et al. (2006, Lemma 3.5). 
Remark 2.4. Recall that the random variables $X_{1}$ and $X_{2}$ are called positively quadrant dependent if

$$
\mathrm{P}\left(X_{1} \leq x_{1}, X_{2} \leq x_{2}\right) \geq \mathrm{P}\left(X_{1} \leq x_{1}\right) \mathrm{P}\left(X_{2} \leq x_{2}\right)
$$

or, equivalently,

$$
\mathrm{P}\left(X_{1}>x_{1}, X_{2}>x_{2}\right) \geq \mathrm{P}\left(X_{1}>x_{1}\right) \mathrm{P}\left(X_{2}>x_{2}\right)
$$

for all $x_{1}$ and $x_{2}$. They are called negatively quadrant dependent if both inequalities above are in the reverse direction. Assumption 2.1 allows both positive and negative quadrant dependence structures to a certain extent. As an example, consider the Ali-Mikhail-Haq copula,

$$
C\left(u_{1}, u_{2} ; \theta\right)=\frac{u_{1} u_{2}}{1-\theta\left(1-u_{1}\right)\left(1-u_{2}\right)}, \quad \theta \in[-1,1),
$$

which is a kind of Archimedean copula with generator $\varphi(z ; \theta)=\ln (1-\theta(1-z)) / z$. This copula can generate both positive and negative quadrant dependence structures depending on the sign of $\theta$. It is easy to verify that Assumption 2.1 is satisfied by this copula.

\subsection{The first main result and its proof}

Now we state our first main result.

Theorem 2.1. Let $X_{1}$ and $X_{2}$ be two random variables with distributions $F_{1}$ and $F_{2}$ concentrated on $[0, \infty)$, respectively, such that Assumption 2.1 holds. Then, the relations

$$
\mathrm{P}\left(S_{2}>x\right) \sim \mathrm{P}\left(X_{(2)}>x\right) \sim \overline{F_{1}}(x)+\overline{F_{2}}(x)
$$

hold for each of the following two cases:

(i) $F_{1} \in \mathcal{S}, F_{2} \in \mathcal{L}$, and $\overline{F_{2}}(x)=O\left(\overline{F_{1}}(x)\right)$;

(ii) $F_{i} \in \mathscr{D} \cap \mathcal{L}$ for $i=1,2$.

A by-product of this theorem is that the distribution of $S_{2}$ belongs to $\&$ for case (i) and belongs to $\mathscr{D} \cap \mathcal{L}$ for case (ii). The key ingredient of our proof of Theorem 2.1 is to bound the probability of $\left(X_{1}, X_{2}\right)$ falling into a trapezoid in the first quadrant above the line $x_{1}+x_{2}=x$. This is motivated by the proof of Lemma 2.7 of Albrecher et al. (2006).

Trivially, the relation $a(x) \sim b(x)$ amounts to the conjunction of $\lim \sup a(x) / b(x) \leq 1$ and $\lim \inf a(x) / b(x) \geq 1$, which are denoted by $a(x) \lesssim b(x)$ and $a(x) \gtrsim b(x)$, respectively. By this and the fact that $S_{2} \geq X_{(2)}$, we see that relations (2.4) follow immediately from Lemmas 2.1 and 2.2, below.

Lemma 2.1. Under the conditions of Theorem 2.1, it holds that

$$
\mathrm{P}\left(S_{2}>x\right) \lesssim \overline{F_{1}}(x)+\overline{F_{2}}(x) .
$$

Proof. We prove Lemma 2.1 under Assumption 2.1 only with $(i, j)=(1,2)$, and we remark that the proof for $(i, j)=(2,1)$ is the same. For arbitrarily fixed $l \geq x_{0}$, we have

$$
\mathrm{P}\left(S_{2}>x\right) \leq \mathrm{P}\left(X_{1}>x\right)+\mathrm{P}\left(X_{2} \geq x-l\right)+\mathrm{P}\left(S_{2}>x, l<X_{1} \leq x\right) .
$$

Since $F_{2}$ is long tailed, $\mathrm{P}\left(X_{2} \geq x-l\right) \sim \overline{F_{2}}(x)$. By Assumption 2.1, there exists some $0<C<\infty$, which does not depend on $l$ or $x$, such that, for all $x \geq l \geq x_{0}$,

$$
\sup _{l<t \leq x} \frac{\mathrm{P}\left(X_{2}>x-t \mid X_{1}=t\right)}{\overline{F_{2}}(x-t)} \leq C .
$$


It follows that

$$
\begin{aligned}
\mathrm{P}\left(S_{2}>x, l<X_{1} \leq x\right) & =\int_{l}^{x} \mathrm{P}\left(X_{2}>x-t \mid X_{1}=t\right) \mathrm{d} F_{1}(t) \\
& \leq C \int_{l}^{x} \overline{F_{2}}(x-t) \mathrm{d} F_{1}(t) \\
& =C\left(\int_{0-}^{\infty} \overline{F_{2}}(x-t) \mathrm{d} F_{1}(t)-\int_{0-}^{l} \overline{F_{2}}(x-t) \mathrm{d} F_{1}(t)-\overline{F_{1}}(x)\right) .
\end{aligned}
$$

The first integral in the bracket is asymptotically equal to $\overline{F_{1}}(x)+\overline{F_{2}}(x)$; for case (i), see Corollary 1 of Cline (1986) while, for case (ii), see Theorem 2.1 of Cai and Tang (2004). Hence, for every $0<\varepsilon<1$ and for all large $x$,

$$
\begin{aligned}
\mathrm{P}\left(S_{2}>x, l<X_{1} \leq x\right) & \leq C\left((1+\varepsilon)\left(\overline{F_{1}}(x)+\overline{F_{2}}(x)\right)-(1-\varepsilon) F_{1}(l) \overline{F_{2}}(x)-\overline{F_{1}}(x)\right) \\
& =C\left(\varepsilon \overline{F_{1}}(x)+\left(\overline{F_{1}}(l)+\varepsilon+\varepsilon F_{1}(l)\right) \overline{F_{2}}(x)\right) .
\end{aligned}
$$

Substituting all these estimates into (2.6) yields, for all large $x$,

$$
\begin{aligned}
\mathrm{P}\left(S_{2}>x\right) & \leq \overline{F_{1}}(x)+(1+\varepsilon) \overline{F_{2}}(x)+C\left(\varepsilon \overline{F_{1}}(x)+\left(\overline{F_{1}}(l)+\varepsilon+\varepsilon F_{1}(l)\right) \overline{F_{2}}(x)\right) \\
& \leq\left(1+\varepsilon+C\left(\overline{F_{1}}(l)+\varepsilon+\varepsilon F_{1}(l)\right)\right)\left(\overline{F_{1}}(x)+\overline{F_{2}}(x)\right) .
\end{aligned}
$$

Relation (2.5) follows because $\varepsilon$ can be arbitrarily small while $l$ can be arbitrarily large.

Lemma 2.2. Let $X_{1}$ and $X_{2}$ be two random variables with distributions $F_{1}$ and $F_{2}$ concentrated on $[0, \infty)$, respectively. Under Assumption 2.1, it holds that

$$
\mathrm{P}\left(X_{(2)}>x\right) \sim \overline{F_{1}}(x)+\overline{F_{2}}(x) .
$$

Proof. Clearly,

$$
\mathrm{P}\left(X_{(2)}>x\right)=\overline{F_{1}}(x)+\overline{F_{2}}(x)-\mathrm{P}\left(X_{1}>x, X_{2}>x\right) .
$$

Following the steps used to derive (2.3), we obtain

$$
\begin{aligned}
\mathrm{P}\left(X_{1}>x, X_{2}>x\right) & =\int_{x}^{\infty} \frac{\mathrm{P}\left(X_{2}>x \mid X_{1}=t\right)}{\mathrm{P}\left(X_{2}>x\right)} \mathrm{P}\left(X_{2}>x\right) \mathrm{d} F_{1}(t) \\
& \leq \overline{F_{1}}(x) \overline{F_{2}}(x) \sup _{x<t<\infty} \frac{\mathrm{P}\left(X_{2}>x+t-t \mid X_{1}=t\right)}{\mathrm{P}\left(X_{2}>x+t-t\right)} \\
& =O(1) \overline{F_{1}}(x) \overline{F_{2}}(x) .
\end{aligned}
$$

This proves relation (2.7).

\section{The multivariate case}

We are going to extend Theorem 2.1 to the multivariate case. For recent references on this topic, we refer the reader to Alink et al. (2004), Barbe et al. (2006), and Kortschak and Albrecher (2008), among others. They all used multivariate copula functions to model the underlying dependence structures.

Let $X_{1}, \ldots, X_{n}$ be $n$ random variables with distributions $F_{1}, \ldots, F_{n}$ concentrated on $[0, \infty)$, respectively, $n \geq 2$. In this case Assumption 2.1 has to be modified to the following. 
Assumption 3.1. There exists some large $x_{0}>0$ such that, for every $j=2, \ldots, n$, the relation

$$
\frac{\mathrm{P}\left(S_{j-1}>x-t \mid X_{j}=t\right)}{\mathrm{P}\left(S_{j-1}>x-t\right)}=O(1)
$$

holds uniformly for all $t \in\left[x_{0}, x\right]$.

Our second main result is given as follows.

Theorem 3.1. Let $X_{1}, \ldots, X_{n}$ be $n$ random variables with distributions $F_{1}, \ldots, F_{n}$ concentrated on $[0, \infty)$, respectively, such that Assumption 3.1 holds for all $j=2, \ldots, n$. Then the relations

$$
\mathrm{P}\left(S_{n}>x\right) \sim \mathrm{P}\left(X_{(n)}>x\right) \sim \sum_{k=1}^{n} \overline{F_{k}}(x)
$$

hold for each of the following two cases:

(i) $F_{k} \in$ s for all $k=1, \ldots, n$, and either $\overline{F_{i}}(x)=O\left(\overline{F_{j}}(x)\right)$ or $\overline{F_{j}}(x)=O\left(\overline{F_{i}}(x)\right)$ for all $i, j=1, \ldots, n$;

(ii) $F_{k} \in \mathcal{D} \cap \mathcal{L}$ for all $k=1, \ldots, n$.

Proof. Clearly,

$$
\mathrm{P}\left(X_{(n)}>x\right) \geq \sum_{k=1}^{n} \overline{F_{k}}(x)-\sum_{1 \leq i<j \leq n} \mathrm{P}\left(X_{i}>x, X_{j}>x\right) .
$$

Under Assumption 3.1, we follow the proof of (2.8) to obtain, for all $1 \leq i<j \leq n$,

$$
\mathrm{P}\left(X_{i}>x, X_{j}>x\right) \leq \mathrm{P}\left(S_{j-1}>x, X_{j}>x\right)=o\left(\overline{F_{j}}(x)\right) .
$$

Therefore,

$$
\mathrm{P}\left(X_{(n)}>x\right) \gtrsim \sum_{k=1}^{n} \overline{F_{k}}(x) .
$$

Since $S_{n} \geq X_{(n)}$, for proving (3.1) it remains to show that

$$
\mathrm{P}\left(S_{n}>x\right) \lesssim \sum_{k=1}^{n} \overline{F_{k}}(x)
$$

We use mathematical induction on $n$. By Lemma 2.1, relation (3.2) holds for $n=2$. Suppose that relation (3.2) holds for $n-1 \geq 2$. Hence, relations (3.1) hold for $n-1$ and the distribution of $S_{n-1}$ belongs to $\&$ for case (i) and belongs to $\mathscr{D} \cap \mathcal{L}$ for case (ii). Furthermore, under the conditions of case (i), either $\mathrm{P}\left(S_{n-1}>x\right)=O\left(\overline{F_{n}}(x)\right)$ or $\overline{F_{n}}(x)=O\left(\mathrm{P}\left(S_{n-1}>x\right)\right)$ holds. Then, by Lemma 2.1 again, it immediately follows that

$$
\mathrm{P}\left(S_{n}>x\right) \lesssim \mathrm{P}\left(S_{n-1}>x\right)+\overline{F_{n}}(x) \sim \sum_{k=1}^{n} \overline{F_{k}}(x) .
$$

Thus, relation (3.2) holds for $n$. 
We give two special cases of Theorem 3.1 to show that Assumption 3.1 is verifiable. Similarly to Remarks 2.2 and 2.3, let the random variables $X_{1}, \ldots, X_{n}$ be dependent according to a multivariate copula function $C\left(u_{1}, \ldots, u_{n}\right)$ and let their distributions $F_{1}, \ldots, F_{n}$ be absolutely continuous and satisfy the conditions of cases (i) or (ii) of Theorem 3.1. Assume that the copula density exists:

$$
C_{1 \cdots n}\left(u_{1}, \ldots, u_{n}\right)=\frac{\partial^{n}}{\partial u_{1} \cdots \partial u_{n}} C\left(u_{1}, \ldots, u_{n}\right) .
$$

Corollary 3.1. Under the above conditions, if, for every nonempty subset I of $\{1, \ldots, n\}$, the marginal copula density $C_{I}\left(u_{i}: i \in I\right)$ is bounded in a neighborhood of the ultimate vertex (whose coordinates are all 1), then Assumption 3.1 is fulfilled. Hence, by Theorem 3.1, relations (3.1) hold.

Proof. Let $c \in(0,1)$, and let $M>0$ be constants such that the inequality

$$
C_{I}\left(u_{i}: i \in I\right) \leq M
$$

holds for every nonempty subset $I$ of $\{1, \ldots, n\}$ and all $u_{i} \in[c, 1]$ with $i \in I$. Let $\tilde{x}>0$ be such that $F_{k}(\tilde{x}) \geq c$ for all $k=1, \ldots, n$ and write $x_{0}=n \tilde{x}$.

With $j=2, \ldots, n$ temporarily fixed, let $I$ be a nonempty subset of $\{1, \ldots, j-1\}$ and write $I^{c}=\{1, \ldots, j-1\} \backslash I$. Note that $C_{I \cup\{j\}}\left(u_{i}: i \in I \cup\{j\}\right)$ represents the copula density corresponding to the random variables $X_{i}$ for $i \in I \cup\{j\}$. We have, for all large $x$ and all $x_{0} \leq t \leq x-x_{0}$,

$$
\begin{aligned}
& \mathrm{P}\left(S_{j-1}>x-t, X_{i}>\tilde{x} \text { for all } i \in I \text { and } X_{i^{c}} \leq \tilde{x} \text { for all } i^{c} \in I^{c} \mid X_{j}=t\right) \\
& \leq \mathrm{P}\left(\sum_{i \in I} X_{i}>x-t-x_{0}, X_{i}>\tilde{x} \text { for all } i \in I \mid X_{j}=t\right) .
\end{aligned}
$$

With $x_{j}=t$, the right-hand side of the above equation is equal to

$$
\int \cdots \int_{\left(\sum_{i \in I} x_{i}>x-t-x_{0}, x_{i}>\tilde{x} \text { for all } i \in I\right)} C_{I \cup\{j\}}\left(F_{i}\left(x_{i}\right): i \in I \cup\{j\}\right) \prod_{i \in I} \mathrm{~d} F_{i}\left(x_{i}\right),
$$

which is further bounded by $M \mathrm{P}\left(\sum_{i \in I} X_{i}^{*}>x-t-x_{0}\right)$, where $\left(X_{1}^{*}, \ldots, X_{n}^{*}\right)$ is an independent copy of $\left(X_{1}, \ldots, X_{n}\right)$. Therefore, uniformly for all $t \in\left[x_{0}, x-x_{0}\right]$,

$$
\begin{aligned}
& \frac{\mathrm{P}\left(S_{j-1}>x-t, X_{i}>\tilde{x} \text { for all } i \in I \text { and } X_{i c} \leq \tilde{x} \text { for all } i^{c} \in I^{c} \mid X_{j}=t\right)}{\mathrm{P}\left(S_{j-1}>x-t\right)} \\
& \quad \leq \frac{M \mathrm{P}\left(\sum_{i \in I} X_{i}^{*}>x-t-x_{0}\right)}{\mathrm{P}\left(S_{j-1}>x-t\right)} \\
& \quad=O(1) \sum_{i \in I} \frac{\mathrm{P}\left(X_{i}>x-t-x_{0}\right)}{\mathrm{P}\left(X_{i}>x-t\right)} \\
& =O(1) .
\end{aligned}
$$

Summing both sides of the above equation over all nonempty subsets $I$ of $\{1, \ldots, j-1\}$ shows that the relation in Assumption 3.1 holds uniformly for all $t \in\left[x_{0}, x-x_{0}\right]$ or, equivalently, uniformly for all $t \in\left[x_{0}, x\right]$. 
Remark 3.1. Clearly, a copula whose joint copula density $C_{1 \ldots n}\left(u_{1}, \ldots, u_{n}\right)$ is uniformly bounded in the whole domain satisfies the requirements in Corollary 3.1. For example, copulas in the Frank family of the form

$$
C\left(u_{1}, \ldots, u_{n} ; \theta\right)=-\frac{1}{\theta}\left(1+\frac{\left(\exp \left(-\theta u_{1}\right)-1\right) \cdots\left(\exp \left(-\theta u_{n}\right)-1\right)}{\left(\mathrm{e}^{-\theta}-1\right)^{n-1}}\right), \quad \theta>0,
$$

as well as copulas in the Clayton family of the form

$$
C\left(u_{1}, \ldots, u_{n} ; \theta\right)=\left(u_{1}^{-\theta}+\cdots+u_{n}^{-\theta}-n+1\right)^{-1 / \theta}, \quad \theta>0,
$$

belong to this category.

Remark 3.2. It might be tempting to generalize the boundedness assumption in Remark 2.3 to the assumption that the joint copula density $C_{1 \ldots n}\left(u_{1}, \ldots, u_{n}\right)$ is uniformly bounded on $[c, 1]^{n}$ for some $0<c<1$. In fact, this is not sufficient. As kindly pointed out to us by a referee, the Gaussian copula $C\left(u_{1}, u_{2}, u_{3}\right)$ with the mean vector $\boldsymbol{\mu}$ and covariance matrix $\boldsymbol{\Sigma}$ given by

$$
\boldsymbol{\mu}=\left[\begin{array}{l}
0 \\
0 \\
0
\end{array}\right], \quad \boldsymbol{\Sigma}=\left[\begin{array}{rrr}
1 & \frac{1}{3} & -\frac{2}{3} \\
\frac{1}{3} & 1 & -\frac{2}{3} \\
-\frac{2}{3} & -\frac{2}{3} & 1
\end{array}\right],
$$

forms such an example. Indeed, we may verify that its joint copula density $C_{123}\left(u_{1}, u_{2}, u_{3}\right)$ is bounded on $\left[\frac{1}{2}, 1\right]^{3}$, but its marginal copula density $C_{12}\left(u_{1}, u_{2}\right)$ is unbounded along the diagonal $u_{1}=u_{2}$ of $\left[\frac{1}{2}, 1\right]^{2}$.

For case (ii) of Theorem 3.1, Assumption 3.1 can be simplified to the following pairwise version.

Assumption 3.2. For each pair $(i, j), 1 \leq i<j \leq n$, and each constant $c>0$, there exists some large $x_{i j}=x_{i j}(c)>0$ such that the relation

$$
\frac{\mathrm{P}\left(c X_{i}>x-t \mid X_{j}=t\right)}{\mathrm{P}\left(c X_{i}>x-t\right)}=O(1)
$$

holds uniformly for all $t \in\left[x_{i j}, x\right]$.

Corollary 3.2. For case (ii) of Theorem 3.1, Assumption 3.2 implies Assumption 3.1. Hence, by Theorem 3.1, relations (3.1) hold under Assumption 3.2.

Proof. With $x_{0}>0$ sufficiently large, it holds, for every $j=2, \ldots, n$, that

$$
\begin{aligned}
\sup _{x_{0} \leq t \leq x} & \frac{\mathrm{P}\left(S_{j-1}>x-t \mid X_{j}=t\right)}{\mathrm{P}\left(S_{j-1}>x-t\right)} \\
\leq & \sup _{x_{0} \leq t \leq x} \sum_{i=1}^{j-1} \frac{\mathrm{P}\left(X_{i}>(x-t) /(j-1) \mid X_{j}=t\right)}{\mathrm{P}\left(X_{i}>x-t\right)} \\
\leq & \sum_{i=1}^{j-1} \sup _{x_{0} \leq t \leq x} \frac{\mathrm{P}\left(X_{i}>(x-t) /(j-1) \mid X_{j}=t\right)}{\overline{F_{i}}((x-t) /(j-1))} \frac{\overline{F_{i}}((x-t) /(j-1))}{\overline{F_{i}}(x-t)} \\
& =O(1),
\end{aligned}
$$

where in the last step we used both Assumption 3.2 and $F_{i} \in \mathcal{D}$. This proves the relation in Assumption 3.1. 


\section{Acknowledgements}

The authors are thankful to Professor Taizhong Hu, Professor Elias Shiu, and an anonymous referee for their helpful comments and constructive suggestions, which have considerably enhanced this work.

\section{References}

Albrecher, H., Asmussen, S. And Kortschak, D. (2006). Tail asymptotics for the sum of two heavy-tailed dependent risks. Extremes 9, 107-130.

Alink, S., LöWE, M. AND WÜTHrich, M. V. (2004). Diversification of aggregate dependent risks. Insurance Math. Econom. 35, 77-95.

Barbe, P., Fougères, A.-L. And Genest, C. (2006). On the tail behavior of sums of dependent risks. Astin Bull. 36, 361-373.

CAI, J. AND TANG, Q. (2004). On max-sum equivalence and convolution closure of heavy-tailed distributions and their applications. J. Appl. Prob. 41, 117-130.

Cline, D. B. H. (1986). Convolution tails, product tails and domains of attraction. Prob. Theory Relat. Fields $\mathbf{7 2 ,}$ 529-557.

Coles, S., Heffernan, J. And Tawn, J. (1999). Dependence measures for extreme value analyses. Extremes 2, 339447.

Embrechts, P., Klüppelberg, C. And Mikosch, T. (1997). Modelling Extremal Events for Insurance and Finance. Springer, Berlin.

Geluk, J. ANd NG, K. (2006). Tail behavior of negatively associated heavy-tailed sums. J. Appl. Prob. 43, 587-593.

KortschaK, D. AND Albrecher, H. (2008). Asymptotic results for the sum of dependent nonidentically distributed random variables. To appear in Methodology Comput. Appl. Prob.

Lehmann, E. L. (1966). Some concepts of dependence. Ann. Math. Statist. 37, 1137-1153.

Nelsen, R. B. (2006). An Introduction to Copulas, 2nd edn. Springer, New York.

TANG, Q. (2008). Insensitivity to negative dependence of asymptotic tail probabilities of sums and maxima of sums. To appear in Stoch. Anal. Appl.

TANG, Q. AND TsitsiashVILI, G. (2003). Randomly weighted sums of subexponential random variables with application to ruin theory. Extremes 6, 171-188. 